\title{
Neural mechanisms for response selection: Comparing selection of responses and items from working memory
}

\author{
Robert Hester, ${ }^{\mathrm{a}, \mathrm{b}, *}$ Mark D’Esposito, ${ }^{\mathrm{c}}$ Michael W. Cole, ${ }^{\mathrm{c}, \mathrm{d}}$ and Hugh Garavan ${ }^{\mathrm{b}}$

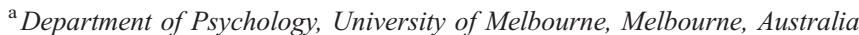 \\ ${ }^{\mathrm{b}}$ Department of Psychology and Trinity College Institute of Neuroscience, Trinity College, Dublin, Ireland \\ ${ }^{\mathrm{c}}$ Helen Wills Neuroscience Institute and Department of Psychology, Henry H. Wheeler Jr Brain Imaging Center, University of California, Berkeley, USA \\ ${ }^{\mathrm{d}}$ Center for the Neural Basis of Cognition, Learning Research and Development Center, and Center for Neuroscience, \\ University of Pittsburgh, Pittsburgh, USA
}

Received 3 January 2006; revised 31 July 2006; accepted 3 August 2006 Available online 30 October 2006

\begin{abstract}
Recent functional imaging studies of working memory (WM) have suggested a relationship between the requirement for response selection and activity in dorsolateral prefrontal (DLPFC) and parietal regions. Although a number of WM operations are likely to occur during response selection, the current study was particularly interested in the contribution of this neural network to WM-based response selection when compared to the selection of an item from a list being maintained in memory, during a verbal learning task. The design manipulated stimulus-response mappings so that selecting an item from memory was not always accompanied with selecting a motor response. Functional activation during selection supported previous findings of fronto-parietal involvement, although in contrast to previous findings left, rather than right, DLPFC activity was significantly more active for selecting a memory-guided motor response, when compared to selecting an item currently maintained in memory or executing a memory-guided response. Our results contribute to the debate over the role of fronto-parietal activity during WM tasks, suggesting that this activity appears particularly related to response selection, potentially supporting the hypothesized role of prefrontal activity in biasing attention toward task-relevant material in more posterior regions. (C) 2006 Elsevier Inc. All rights reserved.
\end{abstract}

\section{Introduction}

The role of persistent neural activity during the retention interval of working memory (WM) tasks has been an enduring interest of cognitive neuroscience, perhaps due to the suggestion that such activity may instantiate how this type of memory is represented in the brain (Fuster, 2000). Although this persistent activity is reported

* Corresponding author. Department of Psychology, School of Behavioural Sciences, University of Melbourne, Victoria 3010, Australia. Fax: +61393476618.

E-mail address: hesterr@unimelb.edu.au (R. Hester).

Available online on ScienceDirect (www.sciencedirect.com). routinely in studies examining WM (Miller et al., 1996; Braver et al., 1997; Klingberg et al., 1997; Cohen et al., 1994; D'Esposito and Postle, 1999; Cabeza and Nyberg, 2000; Owen et al., 2000; Petrides, 2000a; Postle et al., 2000; Rypma et al., 2002), it remains relatively unclear what this activity represents. Rowe and colleagues (Rowe and Passingham, 2001; Rowe et al., 2000) have provided evidence to suggest that selection between items held in WM accounts for activity in area 46 of the prefrontal cortex (PFC), whereas passive maintenance is associated with activation in prefrontal area 8 and intraparietal cortex. Selection is considered the voluntary focusing of attention on a relevant stimulus, consistent with theories that propose the role of the PFC is to competitively bias information processing in more posterior or subcortical regions (Kimberg and Farah, 1993; Duncan and Owen, 2000; Miller and Cohen, 2001).

Although evidence has accumulated to support the argument that response selection is mediated by prefrontal activity (Pochon et al., 2001; Schumacher and D'Esposito, 2002; Schumacher et al., 2003), the studies that have examined selection of a WM-guided response have been unable to control for the contribution that motor planning and production have made to these findings (Curtis and D'Esposito, 2003). For example, Pochon et al. (2001) showed that preparation for an upcoming memory-guided sequence of motor actions relies heavily on PFC, whereas maintaining the same amount of visuospatial information for a yes/no matching task did not. Furthermore, Curtis et al. (2004) showed that preparing an upcoming memory-guided saccade also resulted in significantly greater prefrontal activity when compared to maintaining a spatial location for a later response selection task. The design of these studies prevented a comparison of memory-guided response selection with the selection of an item maintained in memory that did not directly infer a motor response. Such a comparison would clarify the importance of motor planning to the pattern of activity seen during the delay period of WM tasks, which may be critical given the findings from both human and monkey studies that prefrontal cortex is particularly important to the selection and 
planning of motor behavior (Fuster, 1990, 1991; Hoshi et al., 2000).

The aim of the current study was to examine the persistent neural activity observed during a verbal working memory task, where the task design provided a comparison of processes such as selection, preparation and or execution of a WM-guided response, so as to clarify the conditions under which neural activity in parietal and prefrontal regions, a network repeatedly demonstrated as active during these processes, is especially critical. The task design manipulated stimulus-response mappings so that selecting an item from memory did not always involve selecting, and consequently preparing, a motor response. During the stable condition response buttons were mapped serially to allow for response preparation during the selection period. For example, the first position in the serial order was mapped to the leftmost response button, the second serial position to the second button from the left and so on. In the variable response condition itemresponse button mappings were randomized so that subjects had to wait for the response screen (which visually displayed the mappings) before being able to decide on the appropriate response. The variable response condition offered a challenging control condition, matching demands for processes such as maintenance and item selection, but differing on the process of interest, selection of a motor response. A mixed block and event-related fMRI design was used to estimate neural activity for stimulus encoding, maintenance, selecting the appropriate item from a list maintained in memory (with and without item-motor response mappings) and the memory-guided motor responses themselves.

\section{Materials and methods}

\section{Subjects}

Thirteen healthy participants ( 7 females) gave informed consent according to procedures approved by the University of California. Participants were pre-screened, and none used any medication with psychoactive, cardiovascular or homeostatic effects. All participants had normal to corrected vision and were right handed.

\section{Behavioral task}

The working memory selection task (Fig. 1) presented a list of five letters for $4 \mathrm{~s}$ (encoding period) for which subjects were required to maintain serial order (maintenance period: 8-12 s). A probe item from the serial memory list was then presented for $2 \mathrm{~s}$ (selection event), followed by a 6- to 10 -s fixation screen (preparation period: $8-12 \mathrm{~s}$ ). The probe item prompted the subject to select from memory the item that had followed the probe in the memory list. This selection was used to make a motor response during the recall screen (recall period: 2 s). The recall screen displayed each of the items from the memory list along with the button that corresponded to it.

Two conditions of the task were presented. During the stable condition response buttons were mapped serially to allow for response preparation during the selection period. For example, the first position in the serial order was mapped to the leftmost response button, the second serial position to the second button from the left and so on. In the variable response condition itemresponse button mappings were randomized so that subjects had to wait for the response screen (which visually displayed the mappings) to decide. The purpose of this manipulation was to influence the level of motor preparation accompanying selection from WM, prior to the actual WM-guided motor response. Probe items were selected from all four of the available memory list positions (position 5 was not available due to the requirement of selecting the 'next item' from the memory list), with the distribution of positions for probes matched across response conditions. Two runs, each being 15 trials in duration, were administered for both of the conditions (the runs were counterbalanced), with an average trial lasting $44 \mathrm{~s}$ (a 16-s rest period concluded each trial). Participants were informed at the beginning of each run which condition they would be receiving.

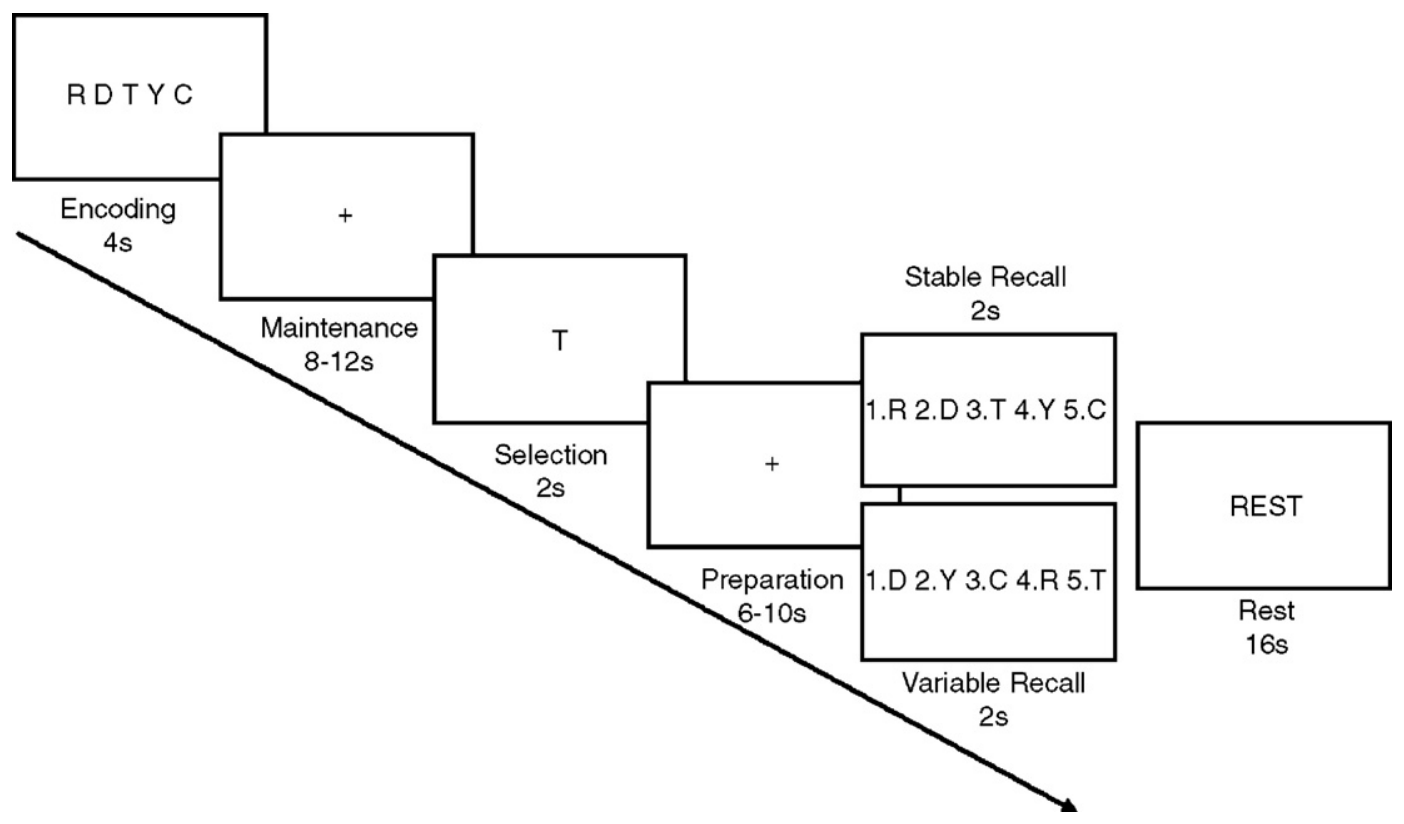

Fig. 1. Working memory selection task. 


\section{Scanning parameters}

Each functional volume contained 18 contiguous $5 \mathrm{~mm}(.5 \mathrm{~mm}$ gap) axial slices covering the entire brain from a 4-T Varian Inova scanner. A gradient-echo, echo-planar pulse sequence $(\mathrm{FOV}=22.4 \mathrm{~cm}$ square field of view; $64 \times 64$ matrix; $3.75 \mathrm{~mm} \times 3.75 \mathrm{~mm}$ in-plane resolution) with a repetition time (TR) of $2 \mathrm{~s}$ was used, though each shot was interpolated with its neighbor resulting in an effective TR of $1 \mathrm{~s}$. High resolution MP-Flash three-dimensional T1-weighted scans were acquired following the functional imaging to allow subsequent activation localization and for spatial normalization. The task was programmed and displayed using E-prime (v.1.1, Psychology Software Tools, Pittsburgh, PA, USA).

\section{Data processing and analyses}

All analyses were conducted using AFNI software v 2.56 (http://afni.nimh.nih.gov/afni/). Following image reconstruction, the time-series data were time-shifted using Fourier interpolation to remove differences in slice acquisition times and motioncorrected using 3-D volume registration (least-squares alignment of three translational and three rotational parameters). Activation outside the brain was also removed using edge detection techniques.

A mixed (block and event-related) analysis was performed to estimate the activation for each part of the task. Separate regressors were created for the block activation periods of encoding, maintenance and preparation. These block regressors were convolved with a standard hemodynamic response to accommodate the lag time of the blood oxygen level-dependant (BOLD) response. The duration of both the maintenance and preparation periods was varied within, and across, trials to assist with the process of separating activity from the different time periods. Condition-specific regressors were derived for all task periods, and trials where participants made recall errors were modeled separately (so as not to confound correct recall activity with error-related activity). A multiple regression analysis was used to determine the average level of block activation as a percentage change relative to the rest period baseline. The baseline activation was derived from averaging the rest periods following each trial.

The multiple regression analysis also calculated event-related impulse response functions (IRFs) for the selection and recall responses. Event-related activity was calculated using a non-linear regression program, which determined the best-fitting gammavariate function for the impulse response functions (IRFs) (Cohen, 1997) as previously described (Murphy and Garavan, 2005). The area under the curve of the gamma-variate function was expressed as a percentage of the area under the baseline. The baseline in this analysis was the average rest period activation from the concluding fixation period of each trial.

The percentage area (event-related activation) and percentage change map (block activation) voxels were re-sampled at $1 \mathrm{~mm}^{3}$ resolution, then warped into standard Talairach space and spatially blurred with a 3-mm isotropic rms Gaussian kernel. Group activation maps for each condition task period (encoding, maintenance, selection, preparation, recall) were determined with one-sample $t$ tests against the null hypothesis of zero event-related activation changes (i.e., no change relative to baseline). Significant voxels passed a voxelwise statistical threshold $(t=4.31, p \leq 0.001)$ and were required to be part of a larger $142 \mu 1$ cluster of contiguous significant voxels. Thresholding was determined through Monte
Carlo simulations and resulted in a $1 \%$ probability of a cluster surviving due to chance.

To compare activation in the stable and variable conditions, thresholded group $t$ test maps were combined to form OR maps. For example, the selection OR map includes the voxels of activation indicated as significant from either the variable or stable selection group activation maps. The same process was performed independently for each task period (encoding, maintenance, selection, preparation and recall). The mean activation for clusters in the OR map was calculated for the purposes of an ROI analysis, and these data were used for a repeated measures comparison between response conditions, corrected using a modified Bonferroni procedure for multiple comparisons (Keppel, 1991).

Behavioral performance data were not available for three subjects included in the fMRI analysis due to technical difficulties during task administration.

\section{Results}

Behavioral performance

High recall accuracy was recorded during both of the response conditions (stable: $97 \pm 0.03 \%$; variable: $93 \pm 0.05 \%, t(9)=2.67$, $p \leq 0.025$ ), with performance consistent across the two runs of each condition [stable: Run $1=97 \%$, Run $2=97 \%$; variable: Run $1=92 \%$, Run2: 95\%]. The attempt to manipulate response preparation appeared to be successful with response times (RTs) for the stable condition $[M=718.4 \mathrm{~s}, S D=232.5]$ significantly faster than during the variable condition $[M=1287.7 \mathrm{~s}, S D=225.7], t(9)=-9.83$, $p \leq 0.001$.

\section{Comparison of functional activation by response condition}

\section{Encoding and maintenance}

Although not of primary interest to the aim of the current study, the encoding and maintenance periods of the task were examined for clusters of significant group activation (see Tables 1 and 2). Stimulus presentation during the encoding and maintenance periods of the task did not differ between the two response conditions. During the encoding period, significant activation was observed in the left frontal (superior and inferior), left temporal, bilateral inferior parietal, posterior cingulate, left putamen and occipital regions. For the maintenance period, significant activation was seen in the left inferior parietal, left fusiform and bilateral occipital regions. Of note was the absence of above-threshold prefrontal activity during the maintenance period (bilateral prefrontal activity was detected at a less conservative threshold, $p<0.005$ ).

\section{Selection and preparation}

During the selection event, subjects were presented with a probe item from the serial working memory list and asked to decide which letter occupied the next serial position. After selecting an item from WM the subject could either prepare to make a memory-guided motor response because of the consistent stimulus-response mapping (stable condition), or they would need to wait for the recall display to present the random stimulusresponse mapping (variable condition). Both conditions would have required selecting the correct WM list item, however the stable condition would appear to have also encouraged the selection of a memory-guided motor-response because of the 
Table 1

Regions of activation during the encoding period of the verbal WM task, for the stable and/or variable conditions

\begin{tabular}{|c|c|c|c|c|c|c|}
\hline \multirow[t]{2}{*}{ Structure } & \multirow[t]{2}{*}{$\mathrm{BA}$} & \multirow[t]{2}{*}{ HS } & \multirow[t]{2}{*}{ Vol $(\mu \mathrm{l})$} & \multicolumn{3}{|c|}{ Center of mass } \\
\hline & & & & $x$ & $y$ & $z$ \\
\hline \multicolumn{7}{|l|}{ Frontal lobe } \\
\hline Superior frontal/pre-SMA & 6 & $\mathrm{~L}$ & 1009 & -3 & 8 & 50 \\
\hline Inferior frontal & 44 & $\mathrm{~L}$ & 381 & -51 & 4 & 23 \\
\hline Precentral & 6 & $\mathrm{~L}$ & 510 & -53 & -1 & 37 \\
\hline \multicolumn{7}{|l|}{ Temporal lobe } \\
\hline Middle & 39 & $\mathrm{~L}$ & 203 & -44 & -59 & 24 \\
\hline \multicolumn{7}{|l|}{ Parietal lobe } \\
\hline \multirow[t]{2}{*}{ Inferior } & 40 & $\mathrm{~L}$ & 1433 & -30 & -49 & 40 \\
\hline & 40 & $\mathrm{R}$ & 167 & 28 & -48 & 43 \\
\hline Angular & 39 & $\mathrm{R}$ & 284 & 43 & -62 & 34 \\
\hline \multirow[t]{3}{*}{ Posterior cingulate } & 30 & $\mathrm{~L}$ & 724 & -2 & -45 & 33 \\
\hline & 30 & $\mathrm{R}$ & 147 & 14 & -62 & 15 \\
\hline & 31 & $\mathrm{R}$ & 145 & 0 & -29 & 38 \\
\hline \multicolumn{7}{|l|}{ Subcortical } \\
\hline Putamen & & $\mathrm{L}$ & 297 & -30 & -3 & 3 \\
\hline \multicolumn{7}{|l|}{ Occipital } \\
\hline Lingual & 18 & $\mathrm{~L}$ & 192 & -1 & -75 & -4 \\
\hline \multicolumn{7}{|l|}{ Cerebellum } \\
\hline Culmen & & $\mathrm{R}$ & 204 & 12 & -53 & -7 \\
\hline Declive & & $\mathrm{R}$ & 189 & 11 & -69 & -11 \\
\hline
\end{tabular}

Positive values for $x, y$ and $z$ Talairach coordinates denote, respectively, locations that are right, anterior and superior relative to the anterior commissure. The table abbreviations indicate the following: BABrodmann area; HS-hemisphere; Vol-activity cluster volume in microliters.

significantly faster RT for this condition. This part of the task was modeled in two parts, a 2-s 'selection' event, and a variable 6- to 10-s 'preparation' block period. A number of regions were significantly active during the selection event (Table 3 and Fig. 2), including several that were not differentiated by condition: bilateral middle frontal (BA 6), posterior cingulate, left occipital and putamen regions. The stable condition demonstrated significantly greater activation in bilateral parietal, left prefrontal (area 9-cluster

Table 2

Regions of activation during the maintenance period of the verbal WM task, for the stable and/or variable conditions

\begin{tabular}{lcccccr}
\hline Structure & BA & HS & Vol $(\mu 1)$ & \multicolumn{3}{c}{ Center of mass } \\
\cline { 5 - 7 } & & & & $x$ & $y$ & $z$ \\
\hline Parietal lobe & & & & & & \\
$\quad$ Inferior parietal & 40 & L & 209 & -42 & -54 & 39 \\
$\quad$ Supramarginal & 40 & L & 331 & -40 & -36 & 35 \\
Temporal lobe & & & & & & \\
$\quad$ Fusiform & 19 & L & 230 & -38 & -65 & -10 \\
& 19 & L & 144 & -32 & -48 & -10 \\
Occipital & & & & & & \\
$\quad$ Middle occipital & 19 & L & 858 & -31 & -81 & 9 \\
$\quad 19$ & $\mathrm{R}$ & 309 & 37 & -74 & 13 \\
$\quad$ Lingual & 18 & $\mathrm{~L}$ & 165 & -12 & -78 & -8 \\
& 18 & $\mathrm{~L}$ & 156 & -21 & -89 & -1 \\
\hline
\end{tabular}

Positive values for $x, y$ and $z$ Talairach coordinates denote, respectively, locations that are right, anterior and superior relative to the anterior commissure. The table abbreviations indicate: BA—Brodmann area; HShemisphere; Vol — activity cluster volume in microliters.
Table 3

Regions of activation during the selection event and preparation period of the verbal WM task, for the stable and/or variable conditions

\begin{tabular}{|c|c|c|c|c|c|c|c|c|}
\hline \multirow[t]{2}{*}{ Structure } & \multirow[t]{2}{*}{ BA } & \multirow[t]{2}{*}{ HS } & \multirow{2}{*}{$\begin{array}{l}\text { Vol } \\
(\mu 1)\end{array}$} & \multicolumn{3}{|c|}{ Center of mass } & \multirow[t]{2}{*}{$p$} & \\
\hline & & & & $x$ & $y$ & $z$ & & \\
\hline \multicolumn{9}{|l|}{ Selection event } \\
\hline \multicolumn{9}{|l|}{ Frontal lobe } \\
\hline \multirow[t]{4}{*}{ Middle frontal } & 6 & $\mathrm{R}$ & 416 & 32 & 1 & 43 & & \\
\hline & 6 & $\mathrm{R}$ & 368 & 27 & 0 & 55 & & \\
\hline & 6 & $\mathrm{~L}$ & 2528 & -31 & -4 & 51 & \multirow[t]{2}{*}{ * } & $\mathrm{S} \Uparrow$ \\
\hline & 6 & $\mathrm{~L}$ & 203 & -41 & 22 & 19 & & \\
\hline Middle/inferior & 9 & $\mathrm{~L}$ & 1013 & -51 & 8 & 28 & * & $\mathrm{S} \Uparrow$ \\
\hline Precentral & 6 & $\mathrm{~L}$ & 268 & -48 & -13 & 33 & & \\
\hline Pre-SMA/ACC & $6 / 32$ & $\mathrm{~L}$ & 4519 & -3 & 10 & 44 & * & $\mathrm{S} \Uparrow$ \\
\hline \multicolumn{9}{|l|}{ Parietal lobe } \\
\hline \multirow[t]{2}{*}{ Inferior parietal } & 40 & $\mathrm{~L}$ & 7496 & -36 & -43 & 43 & * & $\mathrm{S} \Uparrow$ \\
\hline & 40 & $\mathrm{R}$ & 6987 & 35 & -47 & 43 & $*$ & $\mathrm{~S} \Uparrow$ \\
\hline \multirow[t]{2}{*}{ Precuneus } & 7 & $\mathrm{~L}$ & 145 & -6 & -70 & 35 & & \\
\hline & 7 & $\mathrm{R}$ & 212 & 23 & -60 & 28 & * & $\mathrm{V} \Uparrow$ \\
\hline Posterior cingulate & $23 / 30$ & $\mathrm{~L}$ & 342 & -3 & -49 & 22 & & \\
\hline Postcentral & 2 & $\mathrm{~L}$ & 236 & -58 & -20 & 33 & * & $\mathrm{S} \Uparrow$ \\
\hline \multicolumn{9}{|l|}{ Subcortical } \\
\hline Lentiform/putamen & & $\mathrm{L}$ & 180 & -31 & -4 & 7 & & \\
\hline \multicolumn{9}{|l|}{ Occipital } \\
\hline Cuneus & 23 & $\mathrm{~L}$ & 167 & -13 & -62 & 7 & & \\
\hline
\end{tabular}

Preparation period

Parietal lobe

$\begin{array}{llllllll}\text { Inferior parietal } & 40 & \mathrm{R} & 531 & 51 & -43 & 44 & \mathrm{~S} \Uparrow\end{array}$ $\begin{array}{llllll}40 & \mathrm{R} & 277 & 42 & -34 & 35\end{array}$

Positive values for $x, y$ and $z$ Talairach coordinates denote, respectively, locations that are right, anterior and superior relative to the anterior commissure. Asterisks represent clusters that showed a significant effect of response condition $(p<0.05$, corrected), with the accompanying letter and arrow representing the condition with greater activity (variable $(\mathrm{V})$ or stable (S)) and direction of activation in comparison to baseline ( negative). The table abbreviations indicate: BA—Brodmann area; HShemisphere; Vol—activity cluster volume in microliters.

included activation in both middle and inferior frontal gyri), preSMA/ACC (BA 6/32) and left postcentral (area 6) regions.

Given the previous research suggesting a critical role for the right prefrontal cortex in response selection, we were interested in the activity detected in this region during the selection period. The results indicated a cluster of significant activation in the middle frontal (area 6) in the selection period activation OR map (the map identifying activity from either the stable or variable conditions). However, activation in this cluster did not significantly differ between the response conditions. Given the interest in laterality effects, we selected functionally defined left $(x:-51, y: 8, z: 28)$ and right $(x: 32, y: 1, z: 43)$ prefrontal ROIs from the selection event map and compared activity within these two ROIs in a $2 \times 2$ repeated measures ANOVA, using laterality (left, right ROIs) and task period (stable selection, variable recall) as the within-subject factors. The stable selection and variable recall periods were the focus of this analysis as these were the periods were response selection from memory (rather than item selection) was hypothesized to occur. The results of this analysis indicated significant main effects for laterality, with left significantly more active than right $(F(1,12)=2.52, p<0.05)$, and task period, where the selection event showed greater activity than the recall $(F(1,12)=16.10$, $p<0.05)$. The interaction between laterality and task period was not 


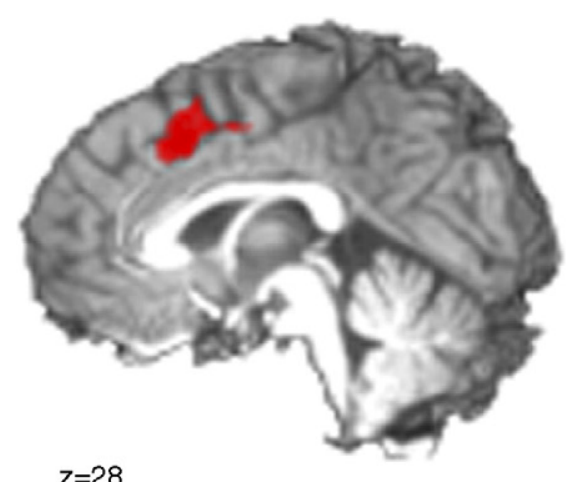

$z=28$
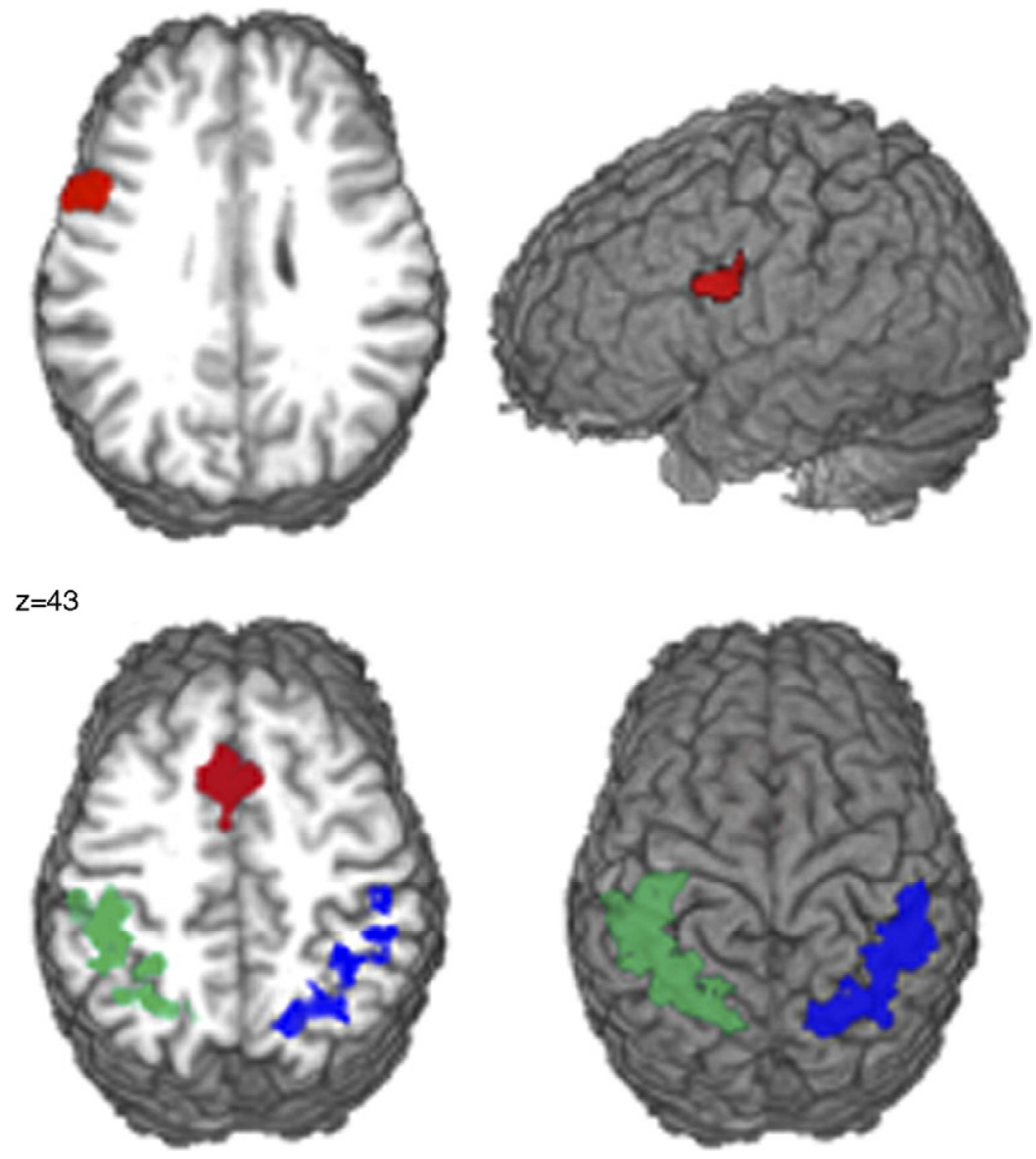

Fig. 2. The left inferior frontal (Brodmann area 9), pre-SMA/ACC (BA 6/32) and bilateral parietal (BA 40) cortical regions that demonstrated significantly greater levels of activation for the stable condition when compared to the variable condition during the selection period of the task.

significant, suggesting that the laterality effect did not vary across task periods.

The preparation period revealed two significant clusters of activity, both in the right inferior parietal lobe. The larger of these two clusters also showed a significant condition effect, with activity during the stable condition significantly higher than during the variable condition.

Recall

The recall period displayed to participants each of the items from the memory list along with the button that corresponded to it.
Whereas both the stable and variable conditions required the participant to provide the correct motor (button press) response, the variable condition required participants to select the response from the random pairings of memory list items with button responses.

Event-related activation during the recall display (Table 4 and Fig. 3) indicated significant activity in a number of regions including: left frontal, anterior and posterior cingulate, bilateral parietal, bilateral occipital, left temporal and subcortical regions (caudate). Significantly greater levels of activity were observed for the variable response condition in regions including the ACC (area 32 and 6), left inferior frontal (area 9), left parietal (large cluster including activation in both superior and inferior gyri), left precentral (area 6) and right thalamus. The left prefrontal region 
Table 4

Regions of event-related activation during the recall period of the verbal WM task, for the stable and/or variable conditions

\begin{tabular}{|c|c|c|c|c|c|c|c|c|}
\hline \multirow[t]{2}{*}{ Structure } & \multirow[t]{2}{*}{ BA } & \multirow[t]{2}{*}{ HS } & \multirow{2}{*}{$\begin{array}{l}\text { Vol } \\
(\mu 1)\end{array}$} & \multicolumn{3}{|c|}{ Center of mass } & \multirow[t]{2}{*}{$p$} & \\
\hline & & & & $x$ & $y$ & $z$ & & \\
\hline \multicolumn{9}{|l|}{ Frontal lobe } \\
\hline Precentral & 6 & $\mathrm{~L}$ & 142 & -35 & -13 & 60 & $*$ & $\mathrm{~V} \Uparrow$ \\
\hline Inferior & 9 & $\mathrm{~L}$ & 170 & -47 & 7 & 28 & $*$ & $\mathrm{~V} \Uparrow$ \\
\hline ACC/Pre-SMA & $32 / 6$ & $\mathrm{~L}$ & 1681 & -1 & 10 & 44 & $*$ & $\mathrm{~V} \Uparrow$ \\
\hline \multicolumn{9}{|l|}{ Parietal lobe } \\
\hline \multirow[t]{2}{*}{ Inferior parietal } & 40 & $\mathrm{R}$ & 804 & 30 & -50 & 46 & & \\
\hline & 40 & $\mathrm{R}$ & 337 & 46 & -27 & 43 & & \\
\hline Superior/inferior & $7 / 40$ & $\mathrm{~L}$ & 1883 & -26 & -55 & 45 & * & $\mathrm{V} \Uparrow$ \\
\hline Posterior cingulate & 30 & $\mathrm{R}$ & 524 & 14 & -64 & 10 & & \\
\hline \multirow[t]{2}{*}{ Postcentral } & 2 & $\mathrm{~L}$ & 325 & -55 & -18 & 18 & & \\
\hline & 2 & $\mathrm{R}$ & 242 & 56 & -8 & 14 & & \\
\hline \multicolumn{9}{|l|}{ Temporal lobe } \\
\hline \multirow[t]{2}{*}{ Fusiform } & 19 & $\mathrm{~L}$ & 569 & -24 & -74 & -11 & & \\
\hline & 19 & $\mathrm{~L}$ & 292 & -41 & -64 & -11 & & \\
\hline Middle temporal & 39 & $\mathrm{~L}$ & 221 & 32 & -37 & -30 & & \\
\hline \multicolumn{9}{|l|}{ Cerebellum } \\
\hline \multirow[t]{2}{*}{ Declive } & & $\mathrm{L}$ & 464 & -32 & -51 & -12 & & \\
\hline & & $\mathrm{R}$ & 210 & 0 & -71 & -9 & & \\
\hline Culmen & & $\mathrm{R}$ & 240 & 4 & -60 & -5 & & \\
\hline \multicolumn{9}{|l|}{ Occipital } \\
\hline \multirow[t]{2}{*}{ Middle occipital } & 18 & $\mathrm{~L}$ & 1327 & -28 & -83 & 6 & & \\
\hline & 18 & $\mathrm{R}$ & 1213 & 25 & 81 & 4 & & \\
\hline \multicolumn{9}{|l|}{ Subcortical } \\
\hline Caudate & & $\mathrm{L}$ & 156 & -28 & -40 & 16 & $*$ & $\mathrm{~V} \Downarrow$ \\
\hline
\end{tabular}

Positive values for $x, y$ and $z$ Talairach coordinates denote, respectively, locations that are right, anterior and superior relative to the anterior commissure. Asterisks represent clusters that showed a significant effect of response condition ( $p<0.05$, corrected), with the accompanying letter and arrow representing the condition with greater activity (variable (V) or stable (S)) and direction of activation in comparison to baseline ( negative). The table abbreviations indicate: BA—Brodmann area; HShemisphere; Vol — activity cluster volume in microliters.

$(x:-47, y: 7, z: 28)$ detected during this condition overlapped with the left prefrontal region $(x:-51, y: 8, z: 28)$ identified during the selection event (see Fig. 4).

Deactivation was detected in the left caudate, with the magnitude of deactivation significantly greater in the variable condition.

Significant activation was not detected in the right prefrontal region.

\section{Discussion}

The persistent neural activity detected during WM tasks has been attributed to a number of cognitive and motor processes. The aim of the current study was to compare the selection of an item and its corresponding motor response from WM, with selection of just the item from WM. When a comparison was made of either selecting an item from a list currently being maintained in memory, or selecting an item that has specific response associated with it (without executing it), greater activity in fronto-cingulate-parietal regions was evident for the latter. Similarly, when the execution of a memory-guided response was compared to the selection and execution of such a response, the latter again showed significantly greater activity in left prefrontal, parietal and pre-SMA/ACC regions. The commonality drawn from these comparisons is that selecting a memory-guided motor response, rather than selecting an item currently maintained in memory or executing a memoryguided response, appears to draw particularly on prefrontal, medial frontal and parietal regions.

These results may offer some clarification of the findings from Rowe and Passingham (2001) who separated the selection and execution of a WM-guided response and showed that when compared to preparing a visually guided response, the magnitude of right prefrontal (area 46 and 9/46) activity was greater. However, they were unable to separate the selection of an item from WM from the selection of a response from WM. Although this is a subtle distinction, the results of the current study suggest that selecting a response from WM recruits left prefrontal activity (located in area 9) to a greater extent than item selection from working memory.

During the recall period of the current task, response selection was required for the variable condition (selecting the motor response that matched a selection-period chosen WM-item), with neither condition requiring item selection. The pattern of activation during the recall period indicated greater levels of left prefrontal activity for the variable condition. The internal consistency of this result across task conditions supports the conclusion that the left prefrontal cortex is critical to selecting a response from WM.

The consistent relationship between left prefrontal activity and response selection from WM contrasts with previous results showing a similar relationship between right prefrontal activity and response selection. The verbal content of the current task may distinguish it from many of the previous studies that also addressed this question. These studies have typically used spatial or oculomotor tasks (Rowe and Passingham, 2001; Rowe et al., 2000; Schumacher and D'Esposito, 2002; Curtis et al., 2004), and comparisons of spatial and nonspatial response selection tasks have suggested that left prefrontal regions may be specifically activated during the latter (Schumacher and Jiang, 2003; Schumacher et al., 2003). The reliance on right-handed responses is unlikely to explain our results, as the aforementioned studies (Rowe and Passingham, 2001; Rowe et al., 2000, 2002) showing that the right prefrontal dominant activation pattern has also used right-handed responses. Past research focused on motor selection, rather than working memory, has found with both transcranial magnetic stimulation and PET techniques that the left hemisphere appears dominant for motor response selection irrespective of hand use (Schluter et al., 1998, 2001). Our results, however, do appear consistent with a recent visuospatial WM study from Volle and colleagues (2005), who found greater left prefrontal activity when a condition requiring maintaining and organizing a WM-based motor response was compared to a similar condition that only required maintenance of the WM material. The consistency of these results across studies using either verbal or visuospatial WM tasks, and the present finding that left prefrontal activity differentiated the response conditions during the selection period despite similar requirements for verbal rehearsal, would suggest that the left prefrontal activity is not attributable to rehearsal strategies.

Increased parietal activation accompanied the greater prefrontal activation seen during response selection, most consistently in the left inferior parietal region, although bilateral parietal activation was seen during the selection event. Although this co-activation has been demonstrated previously (Thompson-Schill et al., 1997; Spence et al., 1998; Rowe and Passingham, 2001; Rowe et al., 

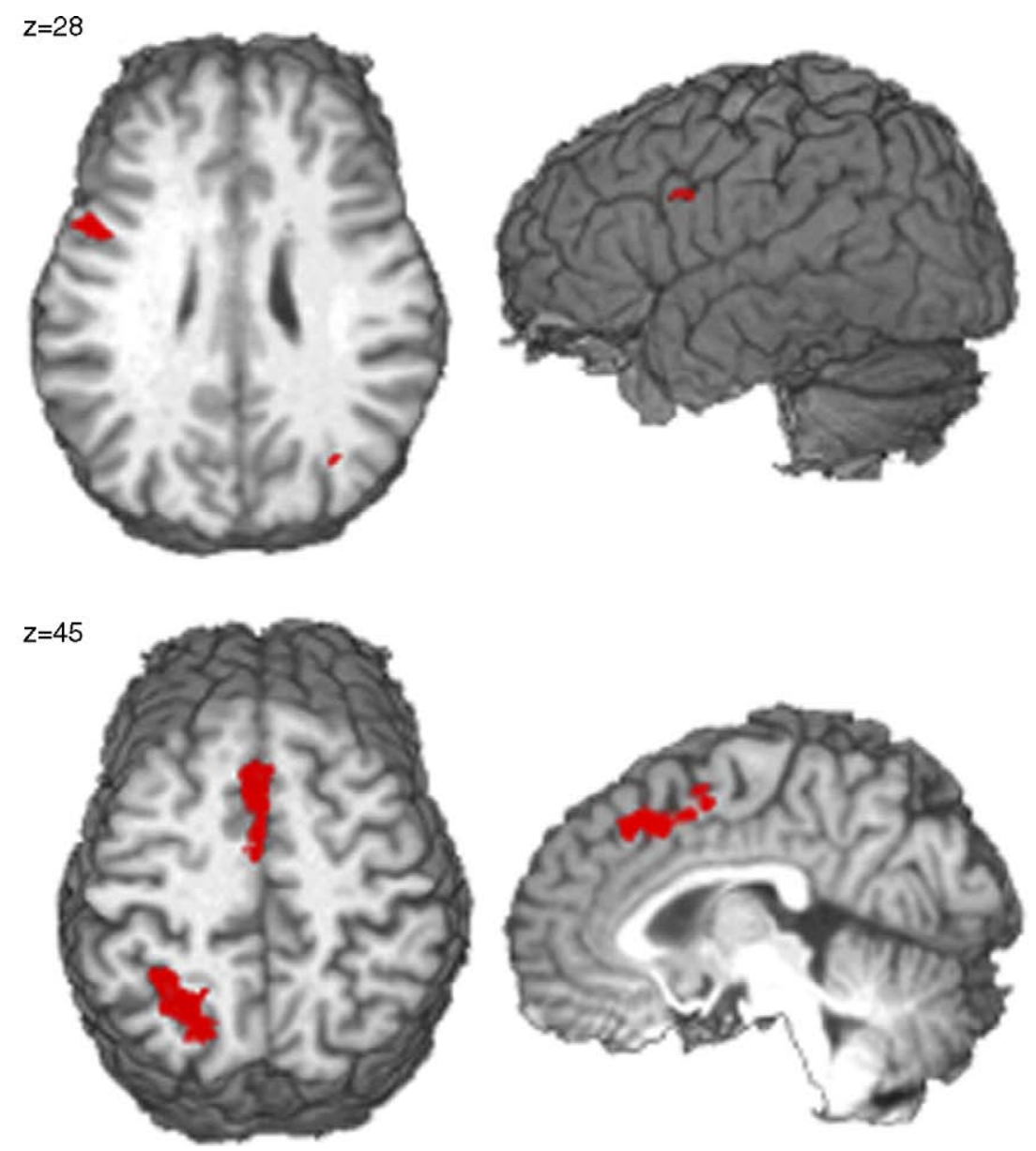

Fig. 3. The left parietal cortical (Brodmann areas 40/7), left inferior frontal (area 9) and pre-SMA/ACC (area 6/32) regions demonstrating significantly greater levels of activation for the variable condition when compared to the stable condition during the recall period of the task.

2004; Bunge et al., 2002), it remains unclear whether it represents a form of reciprocal representation, as Rowe et al. (2004) highlight a lack of uniformity about the exact location of activity (e.g., superior, inferior, medial and intra-parietal regions). It has been proposed that the activity in prefrontal areas 'enhances' the representations of material held in parietal regions (Kimberg and Farah, 1993; Duncan and Owen, 2000; Petrides, 2000b; Miller and Cohen, 2001; Rowe and Passingham, 2001; Bunge et al., 2002; Curtis and D'Esposito, 2003), though the exact nature of what is held in parietal cortices varies between authors.

For example, the current results are consistent with prefrontal activity enhancing a stimulus-response (S-R) association held in the parietal cortex (Miller and Cohen, 2001; Curtis and D'Esposito, 2003), or potentially representing 'motor attention' to right hand motor movements (Rushworth et al., 2001). Greater right inferior parietal activity during the stable condition of the preparation period also appears consistent with the suggestion that this activity is due to maintaining $\mathrm{S}-\mathrm{R}$ associations, rather than verbal material alone (Bunge et al., 2002). The latter appears unlikely as the number of verbal items to remember did not differ between the two response conditions, whereas the S-R association existed only during the stable condition. Evidence from both the animal and human literatures supports the role of the parietal cortex in maintaining 'motor intentions' or 'movement representations' (Toni et al., 2001; Thoenissen et al., 2002; de Lange et al., 2005; Genovesio et al.,

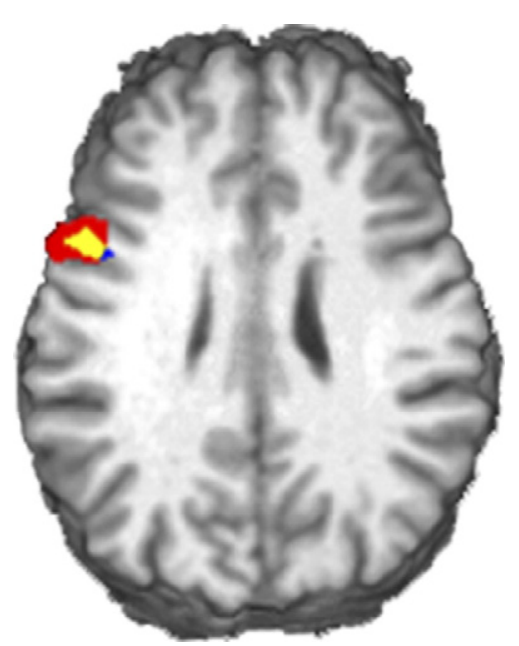

Fig. 4. A figure demonstrating the physical overlap (in yellow) of the left inferior frontal regions identified as showing significantly greater activation for the stable condition of the selection period (red cluster: Brodmann area 9; $x:-51, y: 8, z: 28)$ and variable condition of the recall period (blue cluster: area $9 ; x:-47, y: 7, z: 28)$ during the working memory task. 
2005; Buch et al., 2006), whereas the dorsal premotor cortex (which includes the inferior frontal region identified here) contributes to the generation of this intention/representation.

If a top-down signal from prefrontal to parietal cortices (to bias attention toward particular information held in parietal cortices) is responsible for the pattern of activation seen here, then the strength of this signal appears biased toward selecting a memory-guided motor response rather than selecting between memory-based items. This proposal is consistent with the findings of a recent structural equation modeling study from Rowe et al. (2004), who identified a pattern of increased effective connectivity between prefrontal cortex and modality specific cortical regions. The exact relationship depended on the nature of the material being selected, for visual properties of a stimulus (i.e., color) increased connectivity was seen with the visual cortex, selecting action properties increased connectivity with motor cortex. It is unclear whether this type of top-down biasing of attention is more important to selection of motor programs, rather than verbal responses for example. Such a comparison has been performed previously in relation to response selection (Jiang and Kanwisher, 2003), revealing lower activation in the posterior intraparietal sulcus and precuneus regions during verbal response selection (when compared to visual-manual selections). Although the present study was not ideally designed to perform such a comparison, it does suggest that the dynamic between prefrontal and parietal cortices can vary depending on the output of response selection. It remains unclear whether selection of a memory-guided motor response differs significantly from an equivalent verbal response in terms of the underlying neural mechanisms involved, including whether one modality requires more top-down control than the other. Behavioral data from the Stroop task indicates that difficulty with response selection does vary considerably between verbal and motor versions (Sharma and McKenna, 1998), though patient (and fMRI) studies do not typically compare verbal and motor response selection performance using comparable tasks (Petrides, 2000b; Hunt and Kingstone, 2004). Given the methodological and analytic difficulties associated with verbalization during fMRI studies, a patient-based comparison may be a useful and enlightening approach to this question.

\section{Acknowledgments}

This research was supported by USPHS grant DA14100 and GCRC M01 RR00058 and ARC grant DP0556602 (R.H.). The assistance of Kevin Murphy and Jason Chan are gratefully acknowledged.

\section{References}

Braver, T.S., Cohen, J.D., Nystrom, L.E., Jonides, J., Smith, E.E., Noll, D.C., 1997. A parametric study of prefrontal cortex involvement in human working memory. NeuroImage 5, 49-62.

Buch, E.R., Brasted, P.J., Wise, S.P., 2006. Comparison of population activity in the dorsal premotor cortex and putamen during the learning of arbitrary visuomotor mappings. Exp. Brain Res. 169, 69-84.

Bunge, S.A., Hazeltine, E., Scanlon, M., Rosen, A., Gabrieli, J.D., 2002. Dissociable contributions of prefrontal and parietal cortices to response selection. NeuroImage 17, 1562-1571.

Cabeza, R., Nyberg, L., 2000. Imaging cognition II: an empirical review of 275 PET and fMRI studies. J. Cogn. Neurosci. 12, 1-47.

Cohen, M.S., 1997. Parametric analysis of fMRI data using linear systems methods. NeuroImage 6, 93-103.
Cohen, J.D., Forman, S.D., Braver, T.S., Casey, B.J., Servan-Schreiber, D., Noll, D.C., 1994. Activation of the prefrontal cortex in a nonspatial working memory task with functional MRI. Hum. Brain Mapp. 1, 293-304.

Curtis, C.E., D'Esposito, M., 2003. Persistent activity in the prefrontal cortex during working memory. Trends Cogn. Sci. 7, 415-423.

Curtis, C.E., Rao, V.Y., D’Esposito, M., 2004. Maintenance of spatial and motor codes during oculomotor delayed response tasks. J. Neurosci. 24, 3944-3952.

de Lange, F.P., Hagoort, P., Toni, I., 2005. Neural topography and content of movement representations. J. Cogn. Neurosci. 17, 97-112.

D’Esposito, M., Postle, B.R., 1999. The dependence of span and delayedresponse performance on prefrontal cortex. Neuropsychologia 37, $1303-1315$.

Duncan, J., Owen, A.M., 2000. Common regions of the human frontal lobe recruited by diverse cognitive demands. Trends Neurosci. 23, 475-483.

Fuster, J.M., 1990. Behavioral electrophysiology of the prefrontal cortex of the primate. Prog. Brain Res. 85, 313-323 (dicussion 323-314).

Fuster, J.M., 1991. The prefrontal cortex and its relation to behavior. Prog. Brain Res. 87, 201-211.

Fuster, J.M., 2000. Prefrontal neurons in networks of executive memory. Brain Res. Bull. 52, 331-336.

Genovesio, A., Brasted, P.J., Mitz, A.R., Wise, S.P., 2005. Prefrontal cortex activity related to abstract response strategies. Neuron 47, 307-320.

Hoshi, E., Shima, K., Tanji, J., 2000. Neuronal activity in the primate prefrontal cortex in the process of motor selection based on two behavioral rules. J. Neurophysiol. 83, 2355-2373.

Hunt, A.R., Kingstone, A., 2004. Multisensory executive functioning. Brain Cogn. 55, 325-327.

Jiang, Y., Kanwisher, N., 2003. Common neural substrates for response selection across modalities and mapping paradigms. J. Cogn. Neurosci. $15,1080-1094$

Keppel, G., 1991. Design and Analysis: A Researcher's Handbook. Prentice Hall - Englewood Cliffs, New Jersey.

Kimberg, D., Farah, M., 1993. A unified account of cognitive impairments following frontal lobe damage: the role of working memory in complex organised behaviour. J. Exp. Psychol.: Learn., Mem. Cogn. 26, 336-358.

Klingberg, T., O' Sullivan, B.T., Roland, P.E., 1997. Bilateral activation of fronto-parietal networks by incrementing demand in a working memory task. Cereb. Cortex 7, 465-471.

Miller, E., Cohen, J.D., 2001. An integrative theory of prefrontal cortex function. Annu. Rev. Neurosci. 24, 167-202.

Miller, E.K., Ericson, C.A., Desimone, R., 1996. Neural mechanisms of visual working memory in prefrontal cortex of the macaque. J. Neurosci. $16,5154-5167$

Murphy, K., Garavan, H., 2005. Deriving the optimal number of events for an event-related fMRI study based on the spatial extent of activation. NeuroImage 27, 771-777.

Owen, A.M., Lee, A.C.H, Williams, E.J., 2000. Dissociating aspects of verbal working memory within the human frontal lobe: further evidence for a "process specific" model of lateral frontal organization. Psychobiology 28, 146-155.

Petrides, M., 2000a. Dissociable roles of mid-dorsolateral prefrontal and anterior inferotemporal cortex in visual working memory. J. Neurosci. 20, 7496-7503.

Petrides, M., 2000b. The role of the mid-dorsolateral prefrontal cortex in working memory. Exp. Brain Res. 133, 44-54.

Pochon, J.B., Levy, R., Poline, J.B., Crozier, S., Lehericy, S., Pillon, B., Deweer, B., Le Bihan, D., Dubois, B., 2001. The role of dorsolateral prefrontal cortex in the preparation of forthcoming actions: an fMRI study. Cereb. Cortex 11, 260-266.

Postle, B.R., Berger, J.S., Taich, A.M., D’Esposito, M., 2000. Activity in human frontal cortex associated with spatial working memory and saccadic behavior. J. Cogn. Neurosci. 12, 2-14.

Rowe, J., Passingham, R., 2001. Working memory for location and time: activity in prefrontal area 46 relates to selection rather than maintenance in memory. NeuroImage $14,77-86$. 
Rowe, J.B., Toni, I., Josephs, O., Frackowiak, R.S., Passingham, R.E., 2000. The prefrontal cortex: response selection or maintenance within working memory? Science 288, 1656-1660.

Rowe, J., Friston, K., Frackowiak, R., Passingham, R., 2002. Attention to action: specific modulation of corticocortical interactions in humans. NeuroImage 17, 988-998.

Rowe, J.B., Stephan, K.E., Friston, K., Frackowiak, R.S., Passingham, R.E., 2004. The prefrontal cortex shows context-specific changes in effective connectivity to motor or visual cortex during the selection of action or colour. Cereb. Cortex 15, 85-95.

Rushworth, M.F., Krams, M., Passingham, R.E., 2001. The attentional role of the left parietal cortex: the distinct lateralization and localization of motor attention in the human brain. J. Cogn. Neurosci. 13, 698-710.

Rypma, B., Berger, J.S., D’Esposito, M., 2002. The influence of workingmemory demand and subject performance on prefrontal cortical activity. J. Cogn. Neurosci. 14, 721-731.

Schluter, N.D., Rushworth, M.F., Passingham, R.E., Mills, K.R., 1998. Temporary interference in human lateral premotor cortex suggests dominance for the selection of movements. A study using transcranial magnetic stimulation. Brain 121 (Pt. 5), 785-799.

Schluter, N.D., Krams, M., Rushworth, M.F., Passingham, R.E., 2001. Cerebral dominance for action in the human brain: the selection of actions. Neuropsychologia 39, 105-113.

Schumacher, E.H., D’Esposito, M., 2002. Neural implementation of response selection in humans as revealed by localized effects of stimulus-response compatibility on brain activation. Hum. Brain Mapp. 17, 193-201.
Schumacher, E.H., Jiang, Y., 2003. Neural mechanisms for response selection: representation specific or modality independent? J. Cogn Neurosci. 15, 1077-1079.

Schumacher, E.H., Elston, P.A., D’Esposito, M., 2003. Neural evidence for representation-specific response selection. J. Cogn. Neurosci. 15, 1111-1121.

Sharma, D., McKenna, F.P., 1998. Differential components of the manual and vocal Stroop tasks. Mem. Cogn. 26, 1033-1040.

Spence, S.A., Hirsch, S.R., Brooks, D.J., Grasby, P.M., 1998. Prefrontal cortex activity in people with schizophrenia and control subjects. Evidence from positron emission tomography for remission of 'hypofrontality' with recovery from acute schizophrenia. Br. J. Psychiatry 172, 316-323.

Thoenissen, D., Zilles, K., Toni, I., 2002. Differential involvement of parietal and precentral regions in movement preparation and motor intention. J. Neurosci. 22, 9024-9034.

Thompson-Schill, S.L., D’Esposito, M., Aguirre, G.K., Farah, M.J., 1997. Role of left inferior prefrontal cortex in retrieval of semantic knowledge: a reevaluation. Proc. Natl. Acad. Sci. U. S. A. 94, 14792-14797.

Toni, I., Thoenissen, D., Zilles, K., 2001. Movement preparation and motor intention. NeuroImage 14, S110-S117.

Volle, E., Pochon, J.B., Lehericy, S., Pillon, B., Dubois, B., Levy, R., 2005. Specific cerebral networks for maintenance and response organization within working memory as evidenced by the 'double delay/double response' paradigm. Cereb. Cortex 15 (7), 1064-1074. 